\title{
Leishmaniasis cutánea localizada
}

\section{Cutaneous leishmaniasis located}

\author{
José Juan Ramón de Jesús Trejo-Acuña, ${ }^{*}$ Gisela Navarrete-Franco, ${ }^{\ddagger}$ \\ Alan Gilberto Ramírez-Valverde, ${ }^{\S}$ Erick Alejandro Jiménez-Herrera"
}

\section{RESUMEN}

Las leishmaniasis son un grupo de enfermedades infecciosas ocasionadas por el protozoario del género Leishmania spp. Las principales formas clínicas son la visceral (también llamada kala-azar), mucocutánea y cutánea. En México se le considera una enfermedad poco frecuente que afecta a población marginal y de bajos recursos. En este artículo se comunica el caso de un paciente de sexo masculino de 16 años de edad, con leishmaniasis cutánea localizada.

Palabras clave: Leishmaniasis, leishmaniasis cutánea, Leishmania spp.

\section{ABSTRACT}

Leishmaniasis is a group of infectious diseases caused by the protozoan Leishmania spp. The main clinical forms are visceral (also called kalaazar), muco-cutaneous and cutaneous. In Mexico, it is considered a neglected disease that affects marginal and low-income populations. In this article we present the case of a 16-year-old male patient with localized cutaneous Leishmaniasis.

Keywords: Leishmaniasis, cutaneous leishmaniasis, Leishmania spp.

\section{INTRODUCCIÓN}

Las leishmaniasis son un grupo de enfermedades infecciosas ocasionadas por el protozoario del género Leishmania spp., el cual tiene la facultad de infectar el sistema reticuloendotelial, especialmente los macrófagos. Leishmania spp. se transmite mediante un vector, siendo los principales los mosquitos del género Phlebotomus y Lutzomyia, con aproximadamente 30 especies documentadas. ${ }^{1}$ El reservorio de este protozoario son los mamíferos salvajes y domésticos, algunas veces de forma asintomática, y otras cursando con manifestaciones clínicas evidentes como es el caso en los humanos. Las principales formas clínicas son la visceral (también llamada kala-azar), la mucocutánea y la cutánea. ${ }^{2}$ En México se considera una enfermedad poco frecuente que afecta a la población marginal y de bajos recursos

\footnotetext{
* Dermatólogo.

₹ Jefa del Departamento de Dermatopatología.

\& Dermatólogo.

" Médico residente del tercer año de Dermatología.
}

Centro Dermatológico «Dr. Ladislao de la Pascua», SSCDMX. que radica en áreas agrícolas, especialmente en sembradíos de cacao, café o árboles chicleros, siendo los hombres entre 15 y 44 años de edad los principalmente afectados. La mayoría de los casos documentados en el país pertenecen a tres áreas geográficas bien delimitadas: región del Golfo (Tabasco, Campeche, Quintana Roo y Yucatán), región del Pacífico (Chiapas, Guerrero, Jalisco, Nayarit, Oaxaca y Sinaloa) y región del Centro (Morelos y Puebla), siendo Tabasco, Quintana Roo, Chiapas y Campeche los estados con mayor prevalencia. La forma cutánea localizada es la más frecuente en $99 \%$ de los casos y las principales especies de Leishmania spp. detectadas en el país asociadas a esta variedad son $L$. mexicana y $L$. braziliensis. ${ }^{3}$

Se resaltan tres puntos importantes indispensables para comprender las implicaciones clínicas de la enfermedad: el agente etiológico, el vector y el reservorio. Respecto al agente etiológico, se trata de un parásito digenético que se manifiesta en dos formas: como promastigote, que es el tipo infectante, el cual se desarrolla

Citar como: Trejo-Acuña JJRJ, Navarrete-Franco G, Ramírez-Valverde AG, JiménezHerrera EA. Leishmaniasis cutánea localizada. Rev Cent Dermatol Pascua. 2020;29 (1): 16-19. https://dx.doi.org/10.35366/96265 
en el tracto digestivo de los insectos transmisores, y el amastigote, que es la forma replicativa, intracelular que se ubica en macrófagos, sitio donde se reproduce. ${ }^{4}$ Los transmisores del parásito se conocen como vectores, los principales son hembras hematófagas de la familia Pyschodidae, siendo Lutzomyia el género más importante en México. ${ }^{3}$ Estos parásitos se encuentran en selvas, bosques y en algunas zonas áridas. Finalmente, los reservorios son animales mamíferos silvestres y cánidos domésticos.

Con respecto al ciclo biológico de Leishmania spp., éste comienza con la picadura del insecto hematófago, inoculando el promastigote (con cada picadura se introducen entre 10 y 200), posteriormente es fagocitado por los macrófagos del huésped, donde pierde su flagelo y se convierte en amastigote. Dentro de los macrófagos vivirá en los fagolisosomas evadiendo la respuesta inmunitaria mediante la producción de ectoenzimas y moléculas aún en estudio, así como la inhibición de óxido nítrico por el macrófago activado. Al persistir en el citoplasma del macrófago comienza una replicación de los amastigotes, los cuales finalmente causan la lisis del macrófago. El impacto de estos eventos celulares y moleculares es lo que da lugar a las manifestaciones clínicas de esta dermatosis. ${ }^{5}$

En lo referente al cuadro clínico, en la leishmaniasis cutánea localizada (LCL) el periodo de incubación es de dos semanas a seis meses aproximadamente. Las lesiones se ubican principalmente en miembros (60\%), siguiendo en frecuencia los pabellones auriculares (30\%), la cara y el tronco. ${ }^{1}$ Por lo regular se considera que los sitios expuestos al aire libre son los más accesibles a dichas picaduras. Inicia con una mancha eritematosa el día de la picadura, que dura hasta dos días. Posteriormente se desarrolla una pápula que dura cuatro días aproximadamente, y después se forma un nódulo, que es la lesión elemental característica de la enfermedad. El nódulo puede durar dos semanas aproximadamente, incrementando su tamaño, y posteriormente se ulcera. Esta úlcera es indolora, redonda de fondo limpio (algunas veces con fibrina en el fondo), de bordes indurados. Finalmente, la lesión tiende a la resolución con la formación de una cicatriz. ${ }^{6}$

En el caso de la leishmaniasis cutánea difusa, el cuadro clínico inicia con una mancha o pápula en la zona de la picadura del insecto, diseminándose los protozoarios por contigüidad, vía linfática o hemática a cualquier parte del cuerpo, con excepción de la piel cabelluda, axilas, palmas y plantas. En esta variedad, las pápulas y nódulos tienden a confluir formando placas de diferentes formas y tamaños con bordes bien definidos. ${ }^{7}$
En la leishmaniasis mucocutánea, la principal topografía se ubica a nivel de cara, específicamente en la cavidad oronasal y faringe, con la presencia de las lesiones previamente descritas asociadas a nódulos con superficie anfractuosa, costras serohemáticas y ulceraciones con sangrado. Este tipo se asocia a enfermos mal tratados, y en casos avanzados puede ocasionar mutilación de tejidos. Generalmente se manifiesta meses o años después de haber cicatrizado la forma cutánea. ${ }^{6}$

El diagnóstico de la enfermedad se basa en el cuadro clínico. Pueden realizarse exámenes de laboratorio de tipo directo como impronta, frotis, cultivo, biopsia y PCR, o por métodos indirectos como la inmunoflourescencia indirecta, ELISA, inmunohistoquímica y la prueba de Montenegro. En síntesis, la observación del protozoario es el método diagnóstico más confiable.

En la leishmaniasis cutánea aguda se observan dos hallazgos histopatológicos: 1. La presencia de histiocitos, linfocitos, células plasmáticas con cuerpos de Russell, que en algunas ocasiones logran formar un granuloma de tipo tuberculoide, y 2 . Se observan amastigotes que invaden los histiocitos, lo cual se conoce como cuerpos de Leishman-Donovan. ${ }^{1,8}$

El tratamiento de la leishmaniasis cutánea es médico y los antimoniales pentavalentes se consideran la elección para esta enfermedad como el antimoniato de meglumina (glucantime) y el estibogluconato de sodio (pentostam). Algunos de los efectos adversos de estos fármacos son la anorexia, náuseas, vómito, malestar general, cefalea y mialgias. En el caso de la LCL se recomienda la aplicación local de antimoniales de forma intralesional, una o dos veces a la semana por un mes. Para las otras formas se prefiere utilizar el tratamiento por vía parenteral. En caso de resistencia a antimoniales, se pueden emplear fármacos de segunda línea como la anfotericina B y la pentamidina. También se han utilizado termocirugía y calor local para tratar las lesiones localizadas. ${ }^{3,9,10}$

\section{CASO CLÍNICO}

Se trata de paciente del sexo masculino, de 16 años de edad, comerciante en mercado, originario de San Juan Lalana, Choapam en Oaxaca, y residente de la Ciudad de México, quien acude por presentar una lesión en la cara, a decir del paciente, de tres meses de evolución, que comenzó a crecer tras el rascado. Acudió con médico general en su lugar de residencia, quien únicamente prescribió antimicótico en crema. Al no observar mejoría, acude al Centro Dermatológico «Dr. Ladislao de la Pascua» para diagnóstico y tratamiento. En su 


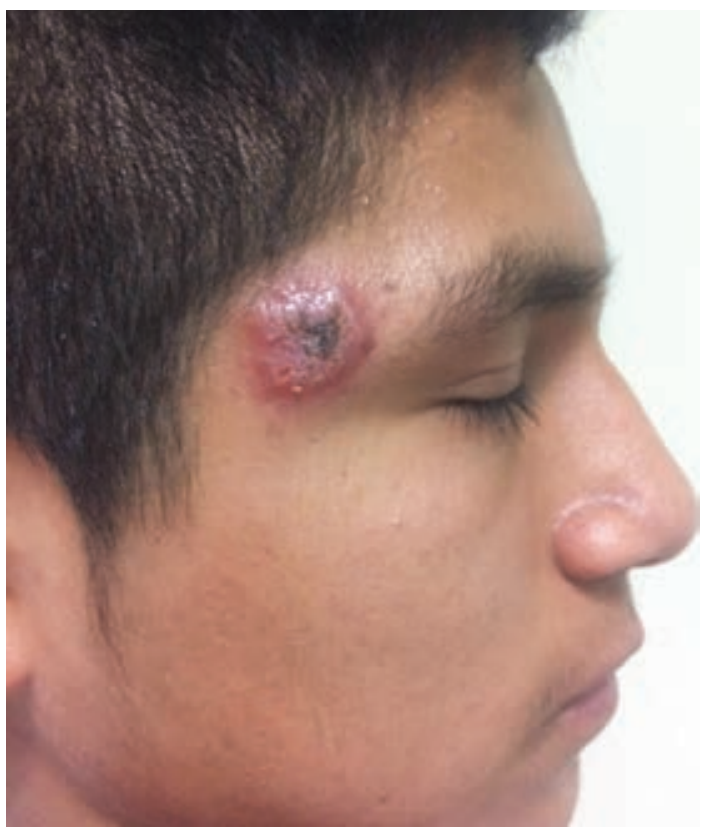

Figura 1: Aspecto general de la dermatosis de tres meses de evolución, sugestiva de leishmaniasis cutánea localizada.

primera consulta se observa una dermatosis localizada a la cabeza, de la que afectaba cara y de ésta, la región frontotemporal derecha, uinilateral. Dicha dermatosis estaba constituida por una neoformación de aspecto nodular, eritematosa, de bordes bien definidos, de $2 \mathrm{~cm}$ de diámetro, con una ulceración central sobre la que se asienta una costra sanguínea (Figura 1). Evolución crónica y asintomática. Resto de la exploración física sin datos patológicos.

Se realiza una biopsia incisional en la que se observa una epidermis con hiperqueratosis con focos de paraqueratosis, depósitos de fibrina, restos celulares y un infiltrado denso que ocupa todo el espesor de la dermis, el cual a mayor aumento muestra histiocitos en cuyo interior se observan cuerpos de inclusión de Leishman-Donovan, rodeados de linfocitos y escasas células plasmáticas. Con todos estos hallazgos se concluye el diagnóstico histopatológico de leishmaniasis (Figuras 2 y 3).

Al no encontrar otras lesiones ni afección sistémica se concluye que se trata de un caso de leishmaniasis cutánea localizada. Se realiza referencia al Centro de Medicina Tropical en el Hospital General de México, donde se le inició manejo a base de antimoniales.

El paciente mostró adecuada evolución con dicho tratamiento, con disminución en el tamaño y la forma de la lesión y, hasta el momento, no se ha reportado algún efecto adverso o eventualidad. Continuará en vigilancia epidemiológica hasta resolución completa de dicha dermatosis conforme a los parámetros de las actuales Guías de Vigilancia Epidemiológica de México.

\section{COMENTARIO}

En el presente caso clínico, el factor que sirvió para orientar el diagnóstico fue el hecho de que el paciente reside en el estado de Oaxaca, donde la prevalencia de este padecimiento es alta, y esta dermatosis debe tomarse en cuenta como posible diagnóstico diferencial ante lesiones de este tipo. La morfología de la lesión era muy característica.

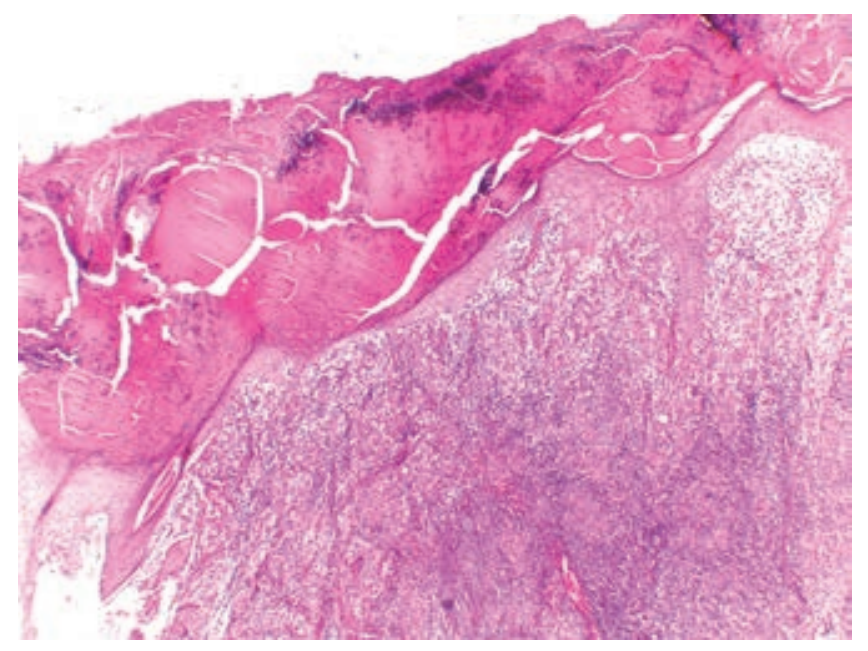

Figura 2: Corte histológico con vista panorámica, tinción (H\&E 10x).

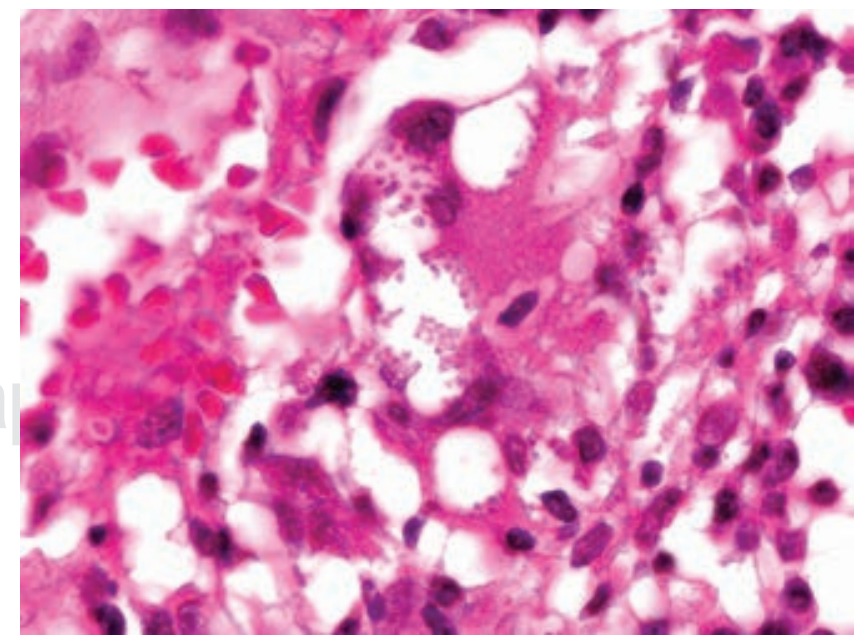

Figura 3: Corte histológico a mayor aumento, en el cual se observan cuerpos de Leishman-Donovan (H\&E 40x). 
Es importante recordar el ciclo del protozoario en el abordaje de un paciente con dichas características clínicas.

Para corroborar este diagnóstico es muy importante la realización de un estudio histopatológico, en el cual se pueden apreciar los cuerpos de LeishmanDonovan, los cuales son los amastigotes dentro de los histiocitos; sin embargo, este hallazgo depende del tiempo de evolución de la enfermedad, pues en las formas crónicas se pueden observar sólo en un bajo porcentaje.

En México aún no se logra la implementación de medidas efectivas para la reducción de la transmisión. Actualmente se opta por la educación del paciente para evitar la picadura del insecto así como la detección oportuna, la búsqueda intencionada de los mismos en zonas endémicas del país, al igual que la vigilancia clínica del tratamiento de casos y su seguimiento conforme las recomendaciones del Programa de Enfermedades Transmitidas por Vector del Centro Nacional de Vigilancia Epidemiológica y Control de Enfermedades.

\section{BIBLIOGRAFÍA}

1. García-Almagro, D. Leishmaniasis cutánea. Actas Dermosifiliogr. 2005; 96: 1-24. doi: 10.1016/S0001-7310(05)73027-1.
2. Pereira A, Pérez M. Leishmaniosis. Parasitología. 2002; 21: 117-124.

3. Manual para el diagnóstico, tratamiento y control de las Leishmaniasis. Centro Nacional de Vigilancia Epidemiológica y Control de Enfermedades. 2015. Recuperado en: http://www.cenaprece. salud.gob.mx/programas/interior/vectores/descargas/pdf/ ManualLeishmaniasis2015.pdf.

4. The Center for food security and public health. Leishmaniasis (cutánea y visceral). 2009; Recovered in: http://www.cfsph.iastate. edu/Diseaselnfo/disease.php?name=leishmaniasis\&lang=es.

5. Scott P. Cutaneous leishmaniasis: immune responses in protection and pathogenesis. Nat Rev Immunol. 2016; 16: 581-592.

6. Torres-Guerrero E, Quintanilla-Cedillo MR, Ruiz-Esmenjaud J, Arenas R. Leishmaniasis: a review. F1000 Res. 2017; 6: 750.

7. Alemayehu, B, Alemayehu M. Leishmaniasis: a review on parasite, vector and reservoir host. Health Sci J. 2017; 11: 1-6.

8. Rosal-Rabes T, Baquero-Artigao F, Miguel G. Leishmaniasis cutánea. Rev Pediatr Atern Primaria. 2010; 12: 263-271.

9. Monzote L. Current treatment of leishmaniasis: a review. The Open Antimicrobial Agents Journal. 2009; 1: 9-19.

10. Savoia D. Recent updates and perspectives on leishmaniasis. J Infect Dev Ctries. 2015; 9: 588-596.

Correspondencia:

Dr. Juan Ramón de Jesús Trejo-Acuña

Dr. Vértiz 464 Esq. Eje 3 Sur,

Col. Buenos Aires, 06780,

Alcaldía Cuauhtémoc, CDMX

Tel: 5556349919.

E-mail: hermesjuanderma@hotmail.com 